\title{
On the Projective Algebra of Randers Metrics of Constant Flag Curvature
}

\author{
Mehdi RAFIE-RAD †† and Bahman REZAEI ${ }^{\S}$ \\ $\dagger$ School of Mathematics, Institute for Research in Fundamental Sciences (IPM), \\ P.O. Box 19395-5746, Tehran, Iran \\ $¥$ Department of Mathematics, Faculty of Mathematical Sciences, University of Mazandaran, \\ P.O. Box 47416-1467, Babolsar, Iran \\ E-mail:rafie-rad@umz.ac.ir,m.rafiei.rad@gmail.com \\ $\S$ Department of Mathematics, Faculty of Sciences, University of Urmia, Urmia, Iran \\ E-mail: b.rezaei@urmia.ac.ir
}

Received February 26, 2011, in final form August 20, 2011; Published online August 31, 2011

http://dx.doi.org/10.3842/SIGMA.2011.085

\begin{abstract}
The collection of all projective vector fields on a Finsler space $(M, F)$ is a finitedimensional Lie algebra with respect to the usual Lie bracket, called the projective algebra denoted by $p(M, F)$ and is the Lie algebra of the projective group $P(M, F)$. The projective algebra $p(M, F=\alpha+\beta)$ of a Randers space is characterized as a certain Lie subalgebra of the projective algebra $p(M, \alpha)$. Certain subgroups of the projective group $P(M, F)$ and their invariants are studied. The projective algebra of Randers metrics of constant flag curvature is studied and it is proved that the dimension of the projective algebra of Randers metrics constant flag curvature on a compact $n$-manifold either equals $n(n+2)$ or at most is $\frac{n(n+1)}{2}$.
\end{abstract}

Key words: Randers metric; constant flag curvature; projective vector field; projective algebra

2010 Mathematics Subject Classification: 53C60; 53B50, 58J60

\section{Introduction}

The motion of freely falling particles define a projective structure on spacetime. Mathematically speaking, this provides a projective connection or an equivalence class of symmetric affine connections all possessing the same unparameterized geodesic curves. This may be regarded as a mathematical formulation of the weak principle of equivalence valid both in the Newtonian and relativistic theory of spacetime and gravity [11]. Many physical considerations require metric structures on spacetime in liaison to affine connections. A necessary condition for two such metric structures to have the same (unparameterized) geodesic curves is that their Weyl projective tensors are identical.

The locally anisotropic space-times are studied in [24] from a geometrical point of view and thus may include some auspices on the Weyl projective tensor. As we will see in this paper, certain subgroups of the Lorentz group may be at once a subgroup of the projective group of the Finsler metric $F=\sqrt{\eta_{\mu \nu} d x^{\mu} d x^{\nu}}+\mathbf{A}_{\mu} d x^{\mu}$, where $\eta$ and $\mathbf{A}$ denotes the Lorentz metric and the electromagnetic potential vector of the flat space-time. Some reduced forms of Weyl projective tensor $W$ have been introduced in $[1,16]$, which are invariant among projectively related constant curvature Finsler metrics but not identical among scalar flag curvature metrics. Any two Finsler metrics possessing the same (unparameterized) geodesics have the same Weyl projective tensor. Studying Weyl projective vector fields (i.e. those vector fields preserving the 
Weyl projective tensor and also the reduced Weyl projective tensors) and projective vector fields have such a leading role to obtain projective symmetries which provide some conservation laws in physical terms. On the other hand, there are many papers devoted on projective symmetry in metric-affine gravity and cosmology, see for example [9, 7].

Randers metrics are the most popular Finsler metrics in differential geometry and physics simply obtained by a Riemannian metric $\alpha=\sqrt{a_{i j}(x) y^{i} y^{j}}$ and $\beta=b_{i}(x) y^{i}$ as $F=\alpha+\beta$ and was introduced by G. Randers in [18] in the context of general relativity. They arise naturally as the geometry of light rays in stationary spacetimes [8]. One may refer to [5, 6, 14, 19] for an extensive series of results about the Einstein Randers metrics and the Randers metrics of constant flag curvature. The present paper is closely related to the problem of projective relatedness of Randers metrics which is investigated in [20]. To avoid the obscurity, given a Randers metric $\alpha+\beta$, the geometric objects in $(M, F)$ and $(M, \alpha)$ are denoted respectively by the prifices " $F-$ " and " $\alpha$-", respectively, for instance, an $F$-projective vector field means a projective vector field on $(M, F)$, an $\alpha$-projective vector field means a projective vector field on $(M, \alpha)$, an $\alpha$-Killing vector fields stands for a Killing vector field on $(M, \alpha)$, etc. We use the usual notations for Randers metrics in $[6,20]$. Given any vector field $V$, its complete lift to $T M_{0}=T M \backslash\{0\}$ is denoted by $\hat{V}$ and the Lie derivative along $\hat{V}$ is denoted by $\mathcal{L}_{\hat{V}}$. One can benefit [28] for an extensive discussion on the theory of Lie derivatives of various geometric objects in Finsler spaces. Traditionally we use the notations for the so-called $(\alpha, \beta)$-metric disposal in [21], thereby $s^{i}{ }_{\circ}=a^{i k} s_{k j} y^{j}$, where $s_{k j}=\left(\frac{\partial b_{k}}{\partial x^{j}}-\frac{\partial b_{j}}{\partial x^{k}}\right)$. We characterize the projective vector fields on a Randers space by proving the following theorem:

Theorem 1. Let $(M, F=\alpha+\beta)$ be a Randers space and $V$ be a vector field on $M . V$ is $F$-projective if and only if $V$ is $\alpha$-projective and $\mathcal{L}_{\hat{V}}\left\{\alpha s^{i}{ }_{0}\right\}=0$.

Determining the dimension of the projective algebra of constant curvature and Einstein spaces is of interests in physical and geometrical discussions, see [7] and interested readers may benefit [28] for a large discussion on this field. This leads to calculate number of independent projective vector fields and is closely related to the number of independent Killing vector fields in each case. It is well known that in an $n$-dimensional Riemannian space of constant curvature the dimension of the projective algebra is $n(n+2)$ and vice-versa, see [7, 28]. This weaves an overture for an analogue problem for Randers space $(M, F=\alpha+\beta)$. If we have $s^{i}{ }_{j}=0$, then the respective projective algebras $p(M, F)$ of $F$ and $p(M, \alpha)$ of $\alpha$ coincide. If moreover, $F$ is locally projectively flat, then $\alpha$ is too and hence, the dimension of the projective algebra $p(M, F)$ is $n(n+2)$. Notice that our discussions are closely related to the algebra $k(M, \alpha)$ of $\alpha$-Killing vector fields. The important case is considerable when $s^{i}{ }_{j} \neq 0$ and uncovers a non-Riemannian feature of Finsler metrics in comparison with the analogue Riemannian case. We summarize the argument by establishing the following result:

Theorem 2. Let $(M, F)$ be an $n$-dimensional $(n \geq 3)$ Randers space of constant flag curvature and $M$ is compact. The dimension of the projective algebra $p(M, F)$ is either $n(n+2)$ or at most equals $\frac{n(n+1)}{2}$.

The spaces admitting certain vector fields has a long history in Riemannian geometry, see for example $[3,7,10,17,25,26,27]$. Existence of some special vector fields on a Riemannian space may pertain to some global properties of the underlying Riemannian space. We prove the following result to uncover such an interaction for Randers spaces:

Theorem 3. Let $(M, F=\alpha+\beta)$ be a Randers space of vanishing $\mathbf{S}$-curvature and dimension $n \geq 3$. If $(M, F)$ admits a non- $\alpha$-affine projective vector field $V$, then $(M, F)$ is a Berwald space. 


\section{Preliminaries}

Let $M$ be a $n$-dimensional $C^{\infty}$ connected manifold. $T_{x} M$ denotes the tangent space of $M$ at $x$. The tangent bundle of $M$ is the union of tangent spaces $T M:=\bigcup_{x \in M} T_{x} M$. We will denote the elements of $T M$ by $(x, y)$ where $y \in T_{x} M$. Let $T M_{0}=T M \backslash\{0\}$. The natural projection $\pi: T M_{0} \rightarrow M$ is given by $\pi(x, y):=x$. A Finsler metric on $M$ is a function $F: T M \rightarrow[0, \infty)$ with the following properties: $(i) F$ is $C^{\infty}$ on $T M_{0},(i i) F$ is positively 1-homogeneous on the fibers of tangent bundle $T M$, and (iii) the Hessian of $F^{2}$ with elements $g_{i j}(x, y):=\frac{1}{2}\left[F^{2}(x, y)\right]_{y^{i} y^{j}}$ is positive definite matrix on $T M_{0}$. The pair $(M, F)$ is then called a Finsler space. Throughout this paper, we denote a Riemannian metric by $\alpha=\sqrt{a_{i j}(x) y^{i} y^{j}}$ and a 1-form by $\beta=b_{i}(x) y^{i}$. A globally defined vector field $\mathbf{G}$ is induced by $F$ on $T M_{0}$, which in a standard coordinate $\left(x^{i}, y^{i}\right)$ for $T M_{0}$ is given by $\mathbf{G}=y^{i} \frac{\partial}{\partial x^{i}}-2 G^{i}(x, y) \frac{\partial}{\partial y^{i}}$, where $G^{i}(x, y)$ are local functions on $T M_{0}$ satisfying $G^{i}(x, \lambda y)=\lambda^{2} G^{i}(x, y), \lambda>0$. Assume the following conventions:

$$
G_{j}^{i}=\frac{\partial G^{i}}{\partial y^{i}}, \quad G^{i}{ }_{j k}=\frac{\partial G_{j}^{i}}{\partial y^{k}}, \quad G_{j k l}^{i}=\frac{\partial G^{i}{ }_{j k}}{\partial y^{l}} .
$$

Notice that the local functions $G^{i}{ }_{j k}$ give rise to a torsion-free connection in $\pi^{*} T M$ called the Berwald connection which is practical in this paper, see [21]. The local functions $G^{i}{ }_{j}$ define a nonlinear connection $\mathcal{H} T M$ spanned by the horizontal frame $\left\{\frac{\delta}{\delta x^{i}}\right\}$, where $\frac{\delta}{\delta x^{j}}=\frac{\partial}{\partial x^{j}}-G^{i}{ }_{j} \frac{\partial}{\partial y^{i}}$. The nonlinear connection $\mathcal{H} T M$ splits $T T M$ as $T T M=\operatorname{ker} \pi_{*} \oplus \mathcal{H} T M$, see [21]. A Finsler metric is called a Berwald metric if $G^{i}{ }_{j k}(x, y)$ are functions of $x$ only at every point $x \in M$, equivalently $F$ is a Berwald metric if and only if $G^{i}{ }_{j k l}=0$.

For a Finsler metric $F$ on an $n$-dimensional manifold $M$ the Busemann-Hausdorff volume form $d V_{F}=\sigma_{F}(x) d x^{1} \cdots d x^{n}$ is defined by

$$
\sigma_{F}(x):=\frac{\operatorname{Vol}\left(\mathbb{B}^{n}(1)\right)}{\operatorname{Vol}\left\{\left(y^{i}\right) \in \mathbb{R}^{n} \mid F\left(\left.y^{i} \frac{\partial}{\partial x^{i}}\right|_{x}\right)<1\right\}} .
$$

Assume $\underline{g}=\operatorname{det}\left(g_{i j}(x, y)\right)$ and define $\tau(x, y):=\ln \frac{\sqrt{g}}{\sigma_{F}(x)} . \quad \tau=\tau(x, y)$ is a scalar function on $T M_{0}$, which is called the distortion [21]. For a vector $y \in T_{x} M$, let $c(t),-\epsilon<t<\epsilon$, denote the geodesic with $c(0)=x$ and $\dot{c}(0)=y$. The function $\mathbf{S}(y):=\frac{d}{d t}[\tau(\dot{c}(t))]_{t=0}$ is called the S-curvature with respect to Busemann-Hausdorff volume form. A Finsler space is said to be of isotropic $\mathbf{S}$-curvature if there is a function $\sigma=\sigma(x)$ defined on $M$ such that $\mathbf{S}=(n+1) \sigma(x) F$. It is called a Finsler space of constant $\mathbf{S}$-curvature once $\sigma$ is a constant. Every Berwald space is of vanishing $\mathbf{S}$-curvature [21]. The $\mathbf{E}$-curvature of the Finsler space $(M, F)$ is defined by $\mathbf{E}_{y}=\mathbf{E}_{i j}(y) d x^{i} \otimes d x^{j}$, where $\mathbf{E}_{i j}=\frac{1}{2} \frac{\partial^{2} \mathbf{S}}{\partial y^{i} \partial y^{j}} .(M, F)$ is called a weakly-Berwald space if $\mathbf{E}=0$. It is easy to see that we have $\mathbf{E}_{i j}=\frac{1}{2} G^{r}{ }_{i r j}$.

Let $(M, \alpha)$ be a Riemannian space and $\beta=b_{i}(x) y^{i}$ be a 1 -form defined on $M$ such that $\|\beta\|_{x}:=\sup _{y \in T_{x} M} \beta(y) / \alpha(y)<1$. The Finsler metric $F=\alpha+\beta$ is called a Randers metric on a manifold $M$. Denote the geodesic spray coefficients of $\alpha$ and $F$ by the notions $G_{\alpha}^{i}$ and $G^{i}$, respectively and the Levi-Civita connection of $\alpha$ by $\nabla$. Define $\nabla_{j} b_{i}$ by $\left(\nabla_{j} b_{i}\right) \theta^{j}:=d b_{i}-b_{j} \theta_{i}{ }^{j}$, where $\theta^{i}:=d x^{i}$ and $\theta_{i}^{j}:=\tilde{\Gamma}_{i k}^{j} d x^{k}$ denote the Levi-Civita connection forms and $\nabla$ denotes its associated covariant derivation of $\alpha$. Let us put

$$
\begin{aligned}
r_{i j} & :=\frac{1}{2}\left(\nabla_{j} b_{i}+\nabla_{i} b_{j}\right), \quad s_{i j}:=\frac{1}{2}\left(\nabla_{j} b_{i}-\nabla_{i} b_{j}\right), \\
s_{j}^{i} & :=a^{i h} s_{h j}, \quad s_{j}:=b_{i} s^{i}{ }_{j}, \quad e_{i j}:=r_{i j}+b_{i} s_{j}+b_{j} s_{i} .
\end{aligned}
$$


Then $G^{i}$ are given by

$$
G^{i}=G_{\alpha}^{i}+\left(\frac{e_{\circ \circ}}{2 F}-s_{\circ}\right) y^{i}+\alpha s_{\circ}^{i},
$$

where $e_{\circ \circ}:=e_{i j} y^{i} y^{j}, s_{\circ}:=s_{i} y^{i}, s^{i}{ }_{\circ}:=s^{i}{ }_{j} y^{j}$ and $G_{\alpha}^{i}$ denote the geodesic coefficients of $\alpha$, see [21]. Notice that the $\mathbf{S}$-curvature of a Randers metric $F=\alpha+\beta$ can be obtained as follows

$$
\mathbf{S}=(n+1)\left\{\frac{e_{\circ \circ}}{F}-s_{\circ}-\rho_{\circ}\right\}
$$

where $\rho=\ln \sqrt{1-\|\beta\|}$ and $\rho_{\circ}=\frac{\partial \rho}{\partial x^{k}} y^{k}$. It is well-known that every weakly-Berwald Randers space is of vanishing $\mathbf{S}$-curvature [21].

Let $F$ be a Finsler metric on an $n$-manifold and $G^{i}$ denote the geodesic coefficients of $F$. Define $\mathbf{R}_{y}=\left.K_{k}^{i}(x, y) d x^{k} \otimes \frac{\partial}{\partial x^{i}}\right|_{x}: T_{x} M \rightarrow T_{x} M$ by

$$
K_{k}^{i}:=2 \frac{\partial G^{i}}{\partial x^{k}}-y^{j} \frac{\partial^{2} G^{i}}{\partial x^{j} \partial y^{k}}+2 G^{j} \frac{\partial^{2} G^{i}}{\partial y^{j} \partial y^{k}}-\frac{\partial G^{i}}{\partial y^{j}} \frac{\partial G^{j}}{\partial y^{k}}
$$

The family $\mathbf{R}:=\left\{\mathbf{R}_{y}\right\}_{y \in T M_{0}}$ is called the Riemann curvature [21]. The Ricci scalar is denoted by Ric it is defined by Ric $:=K_{k}^{k}$. The Ricci scalar Ric is a generalization of the Ricci tensor in Riemannian geometry. A Finsler space $(M, F)$ is called an Einstein space if there is function $\sigma$ defined on $M$ such that Ric $=\sigma(x) F^{2}$. D. Bao and C. Robles proved in [5, 19] the following theorem:

Theorem 4. Let $(M, F=\alpha+\beta)$ be an $n$-dimensional Randers space and $n \geq 3$. If $(M, F)$ is an Einstein space with $\mathbf{R i c}=(n-1) K(x) F^{2}$, then it is of constant $\mathbf{S}$-curvature and $K(x)$ is constant.

The Berwald-Riemannian curvature tensor $\mathbf{K}_{y}=K^{i}{ }_{j k l}(y) \frac{\partial}{\partial x^{i}} \otimes d x^{j} \otimes d x^{k} \otimes d x^{l}$ and the Berwald-Ricci tensor $K_{j l}(y) d x^{j} \otimes d x^{l}$ are respectively defined by

$$
K_{j k l}^{i}:=\frac{1}{3}\left\{\frac{\partial^{2} K_{k}^{i}}{\partial y^{j} \partial y^{l}}-\frac{\partial^{2} K_{l}^{i}}{\partial y^{j} \partial y^{k}}\right\}, \quad K_{j l}:=K^{i}{ }_{j i l} .
$$

Due to a result in [14], every Finsler metric of constant $\mathbf{S}$-curvature on a compact manifold is of vanishing $\mathbf{S}$-curvature. Therefore, the $\mathbf{S}$-curvature of every Einstein Randers metric on an $n$-dimensional $(n \geq 3)$ compact manifold is vanishing.

Denote the horizontal and vertical covariant derivation of the Berwald connection of $F$ respectively by "|" and ".". The quit nouvelle non-Riemannian quantity $\mathbf{H}_{y}=\mathbf{H}_{i j}(y) d x^{i} \otimes d x^{j}$ is simply defined by $\mathbf{H}_{i j}=\mathbf{E}_{i j \mid k} y^{k}$, see $[2,13,15]$. Consider the following Bianchi identity for the Berwald connection [2]:

$$
G^{i}{ }_{j k l \mid m}-G^{i}{ }_{j k m \mid l}=K^{i}{ }_{j k l . m} .
$$

After convecting the indices $i$ and $k$ and taking into account the equation $G^{i}{ }_{j i l}=2 \mathbf{E}_{j l}$, we obtain $G^{k}{ }_{j k l \mid m}-G^{k}{ }_{j k m \mid l}=2\left(\mathbf{E}_{j l \mid m}-\mathbf{E}_{j m \mid l}\right)=K_{j l . m}$. From which it results

$$
y^{j} K_{j l . m}=0, \quad y^{l} K_{j l . m}=-2 \mathbf{H}_{j m} .
$$




\section{$3 \quad$ Projectively related metrics and projectively invariants}

Two Finsler metrics $F$ and $\tilde{F}$ on a manifold $M$ are said to be (pointwise) projectively related if they have the same geodesics as point sets. Hereby, there is a function $P(x, y)$ defined on $T M_{0}$ such that $\tilde{G}^{i}=G^{i}+P y^{i}$ on coordinates $\left(x^{i}, y^{i}\right)$ on $T M_{0}$, where $\tilde{G}^{i}$ and $G^{i}$ are the geodesic spray coefficients of $\tilde{F}$ and $F$, respectively. A Finsler metric $F$ on an open subset $U \subseteq \mathbb{R}^{n}$ is called projectively flat if all geodesics are straight in $U$. In this case, $F$ and the Euclidean metric on $U$ are projectively related. A Finsler metric is called locally projectively flat if at any point $x \in M$, there is a local coordinate $\left(x^{i}, U\right)$ in which $F$ is projectively flat. We consider projectively related Finsler metrics, namely those having the same geodesics as set points. Let $\tilde{F}$ and $F$ be two projectively related Finsler metrics. Consider a natural coordinate system $\left(\left(x^{i}, y^{i}\right), \pi^{-1}(U)\right)$. There is function $P$ defined on $T M_{0}$ such that $\widetilde{G}^{i}=G^{i}+P y^{i}$. Let us put $P_{i}=P_{. i}$ and $P_{i j}=P_{i . j}$. Observe that we have

$$
\begin{aligned}
\widetilde{G}^{i}{ }_{j} & =G^{i}{ }_{j}+P_{j} y^{i}+P \delta^{i}{ }_{j}, \quad \widetilde{G}^{i}{ }_{j k}=G^{i}{ }_{j k}+P_{j k} y^{i}+P_{k} \delta^{i}{ }_{j}+P_{j} \delta^{i}{ }_{k}, \\
\widetilde{\mathbf{E}}_{i j} & =\mathbf{E}_{i j}+\frac{(n+1)}{2} P_{i j} .
\end{aligned}
$$

The Berwald-Riemannian curvature and the Berwald-Ricci tensors of $\tilde{F}$ and $F$ are related as follows

$$
\begin{aligned}
\widetilde{K}_{h j k}^{i}= & K_{h j k}^{i}+y^{i}\left(P_{j h \mid k}-P_{k h \mid j}\right)+\delta_{h}^{i}\left(P_{j \mid k}-P_{k \mid j}\right) \\
& +\delta_{j}^{i}\left(P_{h \mid k}-P_{h} P_{k}-P P_{h k}\right)-\delta_{k}^{i}\left(P_{h \mid j}-P_{h} P_{j}-P P_{h j}\right), \\
\widetilde{K}_{h k}= & K_{h k}+\left(P_{h \mid k}-P_{k \mid h}\right)+(n-1)\left(P_{h \mid k}-P_{h} P_{k}-P P_{h k}\right)-P_{h k \mid \circ} .
\end{aligned}
$$

Finally we find out that $\left(\widetilde{K}_{h k}-\widetilde{K}_{k h}\right)_{. j}=\left(K_{h k}-K_{k h}\right)_{. j}+(n+1)\left(P_{h \mid k}-P_{k \mid h}\right)_{. j}$. The nonRiemannian quantity $\mathbf{H}$ was introduced in [1, 15] and developed in [13]. We would like to consider projectively related Finsler metrics with the same $\mathbf{E}$ - and $\mathbf{H}$-curvatures. Observe that according to (2) and (3), $\mathbf{H}$-curvatures of $\tilde{F}$ and $F$ are related as follows

$$
\begin{aligned}
\widetilde{\mathbf{H}}_{i j} & =y^{r} \frac{\tilde{\delta}}{\tilde{\delta} x^{r}} \widetilde{\mathbf{E}}_{i j}-\widetilde{\mathbf{E}}_{r j} \widetilde{G}_{i}^{r}-\widetilde{\mathbf{E}}_{i r} \widetilde{G}^{r}{ }_{j}=\left(y^{r} \frac{\delta}{\delta x^{r}}-2 P y^{r} \frac{\partial}{\partial y^{r}}\right)\left(\mathbf{E}_{i j}+\frac{(n+1)}{2} P_{i j}\right) \\
& -\left(\mathbf{E}_{r j}+\frac{(n+1)}{2} P_{r j}\right)\left(G^{r}{ }_{i}+P_{i} y^{r}+P \delta^{r}{ }_{i}\right)-\left(\mathbf{E}_{i r}+\frac{(n+1)}{2} P_{i r}\right)\left(G^{r}{ }_{j}+P_{j} y^{r}+P \delta^{r}{ }_{j}\right) \\
& =\mathbf{E}_{i j \mid \circ}+\frac{(n+1)}{2} P_{i j \mid \circ}=\mathbf{H}_{i j}+\frac{(n+1)}{2} P_{i j \mid \circ},
\end{aligned}
$$

where $\frac{\tilde{\delta}}{\tilde{\delta} x^{k}}=\frac{\partial}{\partial x^{k}}-2 \widetilde{G}^{i}{ }_{k} \frac{\partial}{\partial y^{i}}$ and $\frac{\delta}{\delta x^{k}}=\frac{\partial}{\partial x^{k}}-2 G^{i}{ }_{k} \frac{\partial}{\partial y^{i}}$. From (5) one may conclude the following lemma.

Lemma 1. Suppose that $\widetilde{F}$ and $F$ are projectively related with projective factor $P$. Then $\widetilde{F}$ and $F$ have the same $\mathbf{H}$-curvature if and only if $P_{i j \mid \circ}=0$.

The Finsler metrics $\widetilde{F}$ and $F$ are said to be $\mathbf{H}$-projectively related if they are projectively related and have the same $\mathbf{H}$-curvature. From (3) it results that if given any $x \in M$ the function $P(x, y)$ is linear with respect to $y$, in other words $P_{i j}=0$, then $F$ and $\widetilde{F}$ are $\mathbf{H}$ projectively related. Hereby the Finsler metrics $\widetilde{F}$ and $F$ are said to be specially projectively related if $P(x, y)$ is linear with respect to $y$.

Example 1. The Funk metric $\Theta$ on the Euclidean unit ball $\mathbb{B}^{n}(1)$ is a Randers metric given by

$$
\Theta(x, y):=\frac{\sqrt{|y|^{2}-\left(|x|^{2}|y|^{2}-\langle x, y\rangle^{2}\right)}}{1-|x|^{2}}+\frac{\langle x, y\rangle}{1-|x|^{2}}, \quad y \in T_{x} B^{n}(1) \simeq \mathbb{R}^{n},
$$


where $\langle$,$\rangle and |$.$| denotes the Euclidean inner product and norm on \mathbb{R}^{n}$, respectively. Given any constant vector $a \in \mathbb{R}^{n}$, the generalized Funk metric $\Theta_{a}$ is given by $\Theta_{a}:=\Theta+d \varphi_{a}$, where $\varphi_{a}=\ln (1+\langle a, x\rangle)+C$ and $C$ is a constant. From the variational point of view this changes the length function by something which depends only on the end-points, not the path between them. One may also refer to [21] to find an analytic proof. $\Theta$ and $\Theta_{a}$ are both projectively flat, $\mathbf{H}$-projectively related of constant $\mathbf{S}$-curvature with $\sigma=\frac{1}{2}$. It not hard to see that the projective factor $P$ is $P=-\frac{1}{2} d \varphi_{a}$.

Example 2. Given any vector $a \in \mathbb{R}^{n}$, define the Finsler metric $F$ on $\mathbb{B}^{n}(1)$ by

$$
F:=(1+\langle a, x\rangle)\left(\Theta+\Theta_{x^{k}} x^{k}\right) .
$$

$F$ is projectively flat with projective factor $P=\Theta$ and $F$ is of constant flag curvature $\mathbf{K}=0$. Thus $F$ and the Euclidean metric on $\mathbb{B}^{n}(1)$ have the same vanishing $\mathbf{H}$-curvature and are $\mathbf{H}$ projectively related. This example is borrowed from [22].

\subsection{Projectively invariants}

Any geometric object which is identical between two projectively related metrics is called a projective invariant. There are many projectively invariant tensors in Finsler geometry such as the Douglas tensor $\mathbf{D}_{y}=D^{i}{ }_{j k l}(y) \frac{\partial}{\partial x^{i}} \otimes d x^{j} \otimes d x^{k} \otimes d x^{l}$ and Weyl curvature $\mathbf{W}_{y}=$ $W_{j k l}^{i}(y) \frac{\partial}{\partial x^{i}} \otimes d x^{j} \otimes d x^{k} \otimes d x^{l}$. However, the notion of the projective connection in Finsler geometry encounters some difficulties to be globally projectively invariant. The tensors $\mathbf{D}$ and $\mathbf{W}$ are defined as follows

$$
\begin{aligned}
D^{i}{ }_{j k l} & =\frac{\partial^{3}}{\partial y^{j} \partial y^{k} \partial y^{l}}\left\{G^{i}-\frac{1}{n+1} G_{m}^{m} y^{i}\right\}, \\
W^{i}{ }_{j k l} & =K^{i}{ }_{j k l}-\frac{1}{n^{2}-1}\left\{\delta^{i}{ }_{j}\left(\hat{K}_{k l}-\hat{K}_{l k}\right)+\left(\delta^{i}{ }_{k} \hat{K}_{j l}-\delta^{i}{ }_{l} \hat{K}_{j k}\right)+y^{i}\left(\hat{K}_{k l}-\hat{K}_{l k}\right) \cdot j\right\},
\end{aligned}
$$

where $\hat{K}_{j k}=n K_{j k}+K_{k j}+y^{r} K_{k r . j}$. In 1986, H. Akbar-Zadeh introduced a tensor which is just invariant by a sub-group of projective transformations, not all of them [2]. In fact, this is a non-Riemannian generalization of Weyl's curvature. It is denoted by $\stackrel{*}{W}^{i}{ }_{j k l}$ and is defined by

$$
\stackrel{*}{W}^{i}{ }_{j k l}=K_{j k l}^{i}-\frac{1}{n^{2}-1}\left\{\delta_{k}^{i}\left(n K_{j l}+K_{l j}\right)-\delta_{l}^{i}\left(n K_{j k}+K_{k j}\right)+(n-1) \delta_{j}^{i}\left(K_{k l}-K_{l k}\right)\right\} .
$$

Assume that $W^{i}{ }_{k}=y^{j} y^{l} W^{i}{ }_{j k l}$. From (1) $\mathbf{W}$ can be written in terms of $\mathbf{H}$-curvature by the following equation

$$
W_{j k l}^{i}=\stackrel{*}{W}^{i}{ }_{j k l}-\frac{2}{n^{2}-1}\left\{\delta^{i}{ }_{l} \mathbf{H}_{j k}-\delta^{i}{ }_{k} \mathbf{H}_{j l}\right\}-\frac{y^{i}}{n+1}\left(K_{k l}-K_{l k}\right)_{. j} .
$$

One may easily check from (4) and (5) that every two specially projectively related metrics $\tilde{F}$ and $F$ have the same tensors $W, \mathbf{H}$ and $\left(K_{h k}-K_{k h}\right)_{. j}$. Observe that from (6) it results that they have the same tensor $\stackrel{*}{W}$. There is the following identity for $W$ given in [1, 21]:

$$
W_{j k l}^{i}=\frac{1}{3}\left\{W_{k . l . j}^{i}-W_{l . k . j}^{i}\right\} .
$$

Theorem 5. Let $(M, F)$ be an $n$-dimensional Finsler manifold $(n \geq 3)$. $\mathbf{W}=0$ if and only if $F$ is of scalar flag curvature. 
Let $\stackrel{*}{W}_{k}^{i}=y^{j} y^{l} \stackrel{*}{W}_{j k l}{ }_{j k l}$. The following theorem is proved in [1], however, we give a modified proof for it.

Theorem 6. Let $(M, F)$ be an $n$-dimensional Finsler manifold $(n \geq 3) . \stackrel{*}{W}=0$ if and only if $F$ is of constant flag curvature.

Proof. From (7), (6) and $y^{l} \mathbf{H}_{j l}=0$, it follows that we have $W_{k}^{i}=\stackrel{*}{W}^{i}{ }_{k}$ and

$$
W_{j k l}^{i}=\frac{1}{3}\left\{\stackrel{*}{W}_{k . l . j}^{i}-\stackrel{*}{W}^{i}{ }_{l . k . j}\right\} .
$$

Now let $\stackrel{*}{W}=0$. It follows immediately that $W=0$ and from Theorem B. that $(M, F)$ is of scalar curvature. But, from (6) it results

$$
\frac{2}{n^{2}-1}\left\{\delta_{l}^{i} \mathbf{H}_{j k}-\delta^{i}{ }_{k} \mathbf{H}_{j l}\right\}+\frac{y^{i}}{n+1}\left(K_{k l}-K_{l k}\right)_{. j}=0
$$

Convecting the index $k$ in $y^{k}$ and applying (1) yields

$$
-\frac{2}{n^{2}-1} y^{i} \mathbf{H}_{j l}+\frac{2}{n+1} y^{i} \mathbf{H}_{j l}=\frac{2(n-2)}{n^{2}-1} y^{i} \mathbf{H}_{j l}=0
$$

and finally $\mathbf{H}_{j l}=0$, since $n \geq 3$. Now, it results that $(M, F)$ is of constant flag curvature, since $\mathbf{H}=0$. Conversely, suppose that $(M, F)$ is of constant flag curvature. Then, $\mathbf{H}=0, K_{k l}=K_{l k}$ and from (6) it follows that $\stackrel{*}{W}=W=0$, since $(M, F)$ is of constant (scalar) flag curvature.

Remark 1. Projectively related Finsler metrics certainly have the same Weyl and Douglas curvatures. In [20], the authors studied projectively related Randers metrics. Their discussion is closely related to the subject of the present paper.

\section{Projective vector fields on Randers spaces}

Every vector field $V$ on $M$ induces naturally a transformation under the following infinitesimal coordinate transformations on $T M,\left(x^{i}, y^{i}\right) \longrightarrow\left(\bar{x}^{i}, \bar{y}^{i}\right)$ given by

$$
\bar{x}^{i}=x^{i}+V^{i} d t, \quad \bar{y}^{i}=y^{i}+y^{k} \frac{\partial V^{i}}{\partial x^{k}} d t .
$$

This leads to the notion of the complete lift $\hat{V}$ (or traditionally denoted by $V^{C}$, see [28]) of $V$ to a vector field on $T M_{0}$ given by

$$
\hat{V}=V^{i} \frac{\partial}{\partial x^{i}}+y^{k} \frac{\partial V^{i}}{\partial x^{k}} \frac{\partial}{\partial y^{i}} .
$$

Since almost every geometric object in Finsler geometry depend on the both points and velocities, the Lie derivatives of such geometric objects should be regarded with respect to $\hat{V}$. One may get familiar to the theory of Lie derivatives in Finsler geometry in [28]. It is a notable remark in the Lie derivative computations that $\mathcal{L}_{\hat{V}} y^{i}=0$ and the differential operators $\mathcal{L}_{\hat{V}}, \frac{\partial}{\partial x^{i}}$ and $\frac{\partial}{\partial y^{i}}$ commute.

A smooth vector field $V$ on $(M, F)$ is called projective if each local flow diffeomorphism associated with $V$ maps geodesics onto geodesics. If $V$ is projective and each such map preserves affine parameters, then $V$ is called affine, otherwise it is said to be proper projective. The collection of all projective vector fields on $M$ is a finite-dimensional Lie algebra, with respect 
to the usual Lie bracket operation on vector fields, called the projective algebra, and is denoted by $p(M, F)$. It is easy to prove that a vector field $V$ on the Finsler space $(M, F)$ is a projective if and only if there is a function $P$ defined on $T M_{0}$ such that

$$
\mathcal{L}_{\hat{V}} G^{i}=P y^{i},
$$

and $V$ is affine if and only if $P=0$. Whence $F$ is Riemannian, the equation (8) is just $\mathcal{L}_{\hat{V}} \Gamma_{j k}^{i}=\omega_{j} \delta^{i}{ }_{k}+\omega_{k} \delta^{i}{ }_{j}$, where $\omega_{j}$ are the components of a globally defined 1-form on $M$ and thus, $P(x, y)=\omega_{i}(x) y^{i}$.

Proof of Theorem 1. Suppose that $V$ is $F$-projective. Hence it preserves the Douglas tensor, i.e. $\mathcal{L}_{\hat{V}} D^{i}{ }_{j k l}=0$. Let us put $T^{i}=\alpha s^{i}$. The sprays $G^{i}$ of $F$ and $\widehat{G}^{i}=G_{\alpha}^{i}+T^{i}$ are projectively related and thus they have the same Douglas tensor, hence

$$
D^{i}{ }_{j k l}=\widehat{D}^{i}{ }_{j k l}=\frac{\partial^{3}}{\partial y^{j} \partial y^{k} \partial y^{l}}\left\{T^{i}-\frac{1}{n+1} T_{y^{m}}^{m} y^{i}\right\}
$$

A simple calculation shows that $T_{m}^{m}=0$. From that we have

$$
\mathcal{L}_{\hat{V}} D^{i}{ }_{j k l}=\mathcal{L}_{\hat{V}} T^{i}{ }_{j . k . l}=\mathcal{L}_{\hat{V}}\left\{\alpha s^{i}{ }\right\}_{. j . k . l}=0 .
$$

Therefore, there are functions $H^{i}(x, y),(i=1,2, \ldots, n)$ quadratic in $y$ such that

$$
\mathcal{L}_{\hat{V}}\left\{\alpha s^{i}{ }_{\circ}\right\}=H^{i}
$$

Let us put $t_{i j}=\mathcal{L}_{\hat{V}} a_{i j}$. Observe that $\mathcal{L}_{\hat{V}}\left\{\alpha s^{i}{ }_{\circ}\right\}=\frac{t_{\circ}}{2 \alpha} s^{i}{ }_{\circ}+\alpha \mathcal{L}_{\hat{V}} s^{i}{ }$. Now the equation (9) can be re-written as follows:

$$
t_{\circ \circ} s_{\circ}+2 \alpha^{2} \mathcal{L}_{\hat{V}} s^{i}{ }_{\circ}=\alpha H^{i} .
$$

Here we emphasis that $\alpha^{2}=a_{i j}(x) y^{i} y^{j}, t_{\circ \circ} s^{i}{ }_{\circ}=\left(t_{i j}(x) s^{i}{ }_{k}(x)\right) y^{i} y^{k} y^{k}$ and $\mathcal{L}_{\hat{V}} s^{i}{ }_{\circ}=\left(\mathcal{L}_{V} s^{i}{ }_{k}\right)(x) y^{k}$ are polynomials in $y^{1}, y^{2}, \ldots, y^{n}$. Hence the left hand of $(10)$ is a polynomial in $y^{1}, y^{2}, \ldots, y^{n}$ for every $i$, while the right hand is not. It follows immediately that $H^{i}=0$ for every index $i$ and (9) reads as $\mathcal{L}_{\hat{V}}\left\{\alpha s^{i}{ }_{\circ}\right\}=0$. Recall that the geodesic coefficients of $F$ are of the following form:

$$
G^{i}=G_{\alpha}^{i}+\left(\frac{e_{\circ \circ}}{2 F}-s_{\circ}\right) y^{i}+\alpha s_{\circ}^{i} .
$$

From $\mathcal{L}_{\hat{V}}\left\{\alpha s^{i}{ }_{o}\right\}=0$ and $\mathcal{L}_{\hat{V}} G^{i}=P y^{i}$ it results now, that we have

$$
\mathcal{L}_{\hat{V}} G^{i}=\mathcal{L}_{\hat{V}}\left\{G_{\alpha}^{i}+\left(\frac{e_{\circ}}{2 F}-s_{\circ}\right) y^{i}\right\}=P y^{i},
$$

and finally we obtain

$$
\mathcal{L}_{\hat{V}} G_{\alpha}^{i}=\left\{P-\mathcal{L}_{\hat{V}}\left(\frac{e_{\circ}}{2 F}-s_{\circ}\right)\right\} y^{i},
$$

which shows that $V$ is a $\alpha$-projective vector field. Conversely suppose that $V$ is $\alpha$-projective (i.e. $\mathcal{L}_{\hat{V}} G_{\alpha}^{i}=\omega_{\circ} y^{i}$, for some 1 -forms $\omega_{\circ}=\omega_{k}(x) y^{k}$ on $M$ ) and $\mathcal{L}_{\hat{V}}\left\{\alpha s^{i}{ }_{\circ}\right\}=0$. From (11) it follows

$$
\begin{aligned}
\mathcal{L}_{\hat{V}} G^{i} & =\mathcal{L}_{\hat{V}}\left\{G_{\alpha}^{i}+\left(\frac{e_{\circ \circ}}{2 F}-s_{\circ}\right) y^{i}+\alpha s_{0}^{i}\right\}=\mathcal{L}_{\hat{V}} G_{\alpha}^{i}+\mathcal{L}_{\hat{V}}\left(\frac{e_{\circ}}{2 F}-s_{\circ}\right) y^{i} \\
& =\left\{\omega_{\circ}+\mathcal{L}_{\hat{V}}\left(\frac{e_{\circ \circ}}{2 F}-s_{\circ}\right)\right\} y^{i}
\end{aligned}
$$

which proves that $V$ is a $F$-projective vector field. 
Let us prove initially the following lemma:

Lemma 2. Let $(M, F=\alpha+\beta)$ be an $n$-dimensional Randers space. If $s^{i}{ }_{j} \neq 0$, then $V$ is $F$ projective vector field if and only if it is a $\alpha$-homothety and $\mathcal{L}_{\hat{V}} d \beta=\mu d \beta$, where $\mathcal{L}_{\hat{V}} a_{i j}=2 \mu a_{i j}$.

Proof. Suppose that $s^{i}{ }_{\circ} \neq 0$. By Theorem $1, V$ is $F$-projective if and only if it is $\alpha$-projective and $\mathcal{L}_{\hat{V}}\left\{\alpha s^{i}{ }{ }\right\}=0$. Let us suppose $t_{i j}=\mathcal{L}_{\hat{V}} a_{i j}$ and observe that $\mathcal{L}_{\hat{V}}\left\{\alpha s^{i}{ }\right\}=0$ is equivalent to

$$
t_{\circ \circ} s_{\circ}{ }_{\circ}+2 \alpha^{2} \mathcal{L}_{\hat{V}} s_{\circ}^{i}=0 .
$$

It follows that $\alpha^{2}$ divides $t_{\circ \circ} s^{i}$ ofor every index $i$. This equivalent to that $s^{i}{ }_{j}=0$ or $\alpha^{2}$ divides $t_{\circ \circ}$ which means that $V$ is a conformal vector field on $(M, \alpha)$, since $s^{i}{ }_{j} \neq 0$. Since $V$ is already $\alpha$ projective, it follows that $V$ is an $\alpha$-homothety and there is a constant $\mu$ such that $\mathcal{L}_{V} a_{i j}=2 \mu a_{i j}$. From (12) we obtain $\mathcal{L}_{V} s^{i}{ }_{j}=-\mu s^{i}{ }_{j}$. Now observe that

$$
\mathcal{L}_{V} s_{i j}=\mathcal{L}_{V}\left\{a_{i k} s^{k}{ }_{j}\right\}=\left(\mathcal{L}_{V} a_{i k}\right) s^{k}{ }_{j}+a_{i k} \mathcal{L}_{V} s^{k}{ }_{j}=2 \mu a_{i k} s^{k}{ }_{j}-\mu a_{i k} s^{k}{ }_{j}=\mu s_{i j} .
$$

Proof of Theorem 2. Let us suppose that $M$ is compact and $F=\alpha+\beta$ is a Randers metric of constant flag curvature and $n \geq 3$. Following [5], $F$ is of constant $\mathbf{S}$-curvature and due to a result about constant $\mathbf{S}$-curvature Finsler spaces in [14], it follows that $\mathbf{S}=0$. This results $e_{\circ \circ}=r_{\circ \circ}+2 \beta s_{\circ}=0$. Now let us suppose that $s_{j}{ }_{j} \neq 0$. By Lemma 2, every $F$-projective vector field $V$ is an $\alpha$-homothety and since $M$ is compact, thus every $F$-projective vector field $V$ is $\alpha$-Killing. Hence in this case we have the inclusion $p(M, F) \subseteq k(M, \alpha)$, where $k(M, \alpha)$ denotes the Lie algebra of $\alpha$-Killing vector fields. It is well-known that the dimension of algebra of $\alpha$-Killing vector fields is at most $\frac{n(n+1)}{2}$. Therefore $\operatorname{dim}(p(M, F)) \leq \frac{n(n+1)}{2}$. Now let us suppose that $s^{i}{ }_{j}=0$. In this case we have $p(M, F)=p(M, \alpha)$ and moreover one conclude that $\nabla_{j} b_{i}=0$ and $F$ is a Berwald metric. Since $F$ is of constant flag curvature, thus $F$ and $\alpha$ are metrics of zero flag curvatures. Notice that $F$ is of constant flag curvature, its Weyl curvature vanishes and since $s^{i}{ }_{j}=0$, thus $F$ and $\alpha$ are projectively related and hence $\alpha$ has vanishing Weyl curvature and by Beltrami's theorem, $\alpha$ has constant sectional curvature. It is well-known that the dimension of $p(M, \alpha)$ is $n(n+2)$. Hence we have $\operatorname{dim}(p(M, F))=n(n+2)$.

The following inclusive result follows from the proof of Theorem 2.

Corollary 1. Let $(M, F=\alpha+\beta)$ be a Randers space of constant flag curvature. The following statements hold:

(a) if $\beta$ is closed, then $p(M, F)=p(M, \alpha)$;

(b) if $\beta$ is not a closed 1 -form, then $p(M, F) \subseteq h(M, \alpha)$, where $h(M, \alpha)$ denotes the Lie algebra of $\alpha$-homothety vector fields.

Proof of Theorem 3. To obtain general formulae, let us assume that $(M, F=\alpha+\beta)$ be a Randers space of isotropic S-curvature $\mathbf{S}=(n+1) \sigma(x) F$ and $V$ be a non-affine projective vector field. Following a result in [23], it results that $e_{\circ \circ}=2 \sigma(x)\left(\alpha^{2}-\beta^{2}\right)$. Suppose that there is a function $\Psi$ such that $\Psi(x, y)$ is linear with respect to $y$ such that $\mathcal{L}_{\hat{V}} G^{i}=\Psi y^{i}$. By applying Theorem 1, we have

$$
\mathcal{L}_{\hat{V}} G^{i}=\mathcal{L}_{\hat{V}} \widetilde{G}^{i}+\mathcal{L}_{\hat{V}}\left(\sigma(\alpha-\beta) y^{i}\right)-\mathcal{L}_{\hat{V}} s_{\circ} y^{i}=\Psi y^{i} .
$$

Put $t_{i j}=\mathcal{L}_{\hat{V}} a_{i j}$. It is well-known that $\mathcal{L}_{\hat{V}} y^{i}=0$, it follows $t_{\circ \circ}=\mathcal{L}_{\hat{V}} \alpha^{2}$ and

$$
\mathcal{L}_{\hat{V}} \widetilde{G}^{i}+\alpha \mathcal{L}_{\hat{V}} \sigma y^{i}-\beta \mathcal{L}_{\hat{V}} \sigma y^{i}+\frac{t_{\circ \circ}}{2 \alpha} c y^{i}-\sigma y^{i} \mathcal{L}_{\hat{V}} \beta-\mathcal{L}_{\hat{V}} s_{\circ} y^{i}=\Psi y^{i}
$$


Given any natural coordinate system $\left(\left(x^{i}, y^{i}\right), \pi^{-1}(U)\right)$ and $x \in U$, we can regard each terms of the above equation as a polynomial in $y^{1}, y^{2}, \ldots, y^{n}$. Multiplying the two sides of the last equation in $\alpha$, we obtain the following relation:

$$
\operatorname{Rat}^{i}+\alpha \operatorname{Irrat}^{i}=0, \quad i=1,2, \ldots, n,
$$

where the polynomials Rat $^{i}$ and $\operatorname{Irrat}^{i}$ are given by

$$
\begin{aligned}
& \operatorname{Rat}^{i}=\alpha^{2} \mathcal{L}_{\hat{V}} \sigma y^{i}+\frac{1}{2} t_{\circ \circ} \sigma y^{i}, \\
& \text { Irrat }^{i}=\mathcal{L}_{\hat{V}} \widetilde{G}^{i}-\left(\beta \mathcal{L}_{\hat{V}} \sigma+\sigma \mathcal{L}_{\hat{V}} \beta+\mathcal{L}_{\hat{V}} s_{\circ}+\Psi\right) y^{i} .
\end{aligned}
$$

Now let us assume $\mathbf{S}=(n+1) \sigma(x) F=0$. By Lemma $2,(M, F)$ must be locally projectively flat, otherwise $V$ is a $\alpha$-homothety which is a contradiction to the assumption that $V$ is non- $\alpha$ homothety. Hence $s_{i j}=0$ and by $e_{i j}=r_{i j}+b_{i} s_{j}+b_{j} s_{i}=r_{i j}=0$. This is equivalent to $\nabla_{i} b_{j}=0$ and $(M, F)$ is a Berwald space.

By Theorem 2, the following theorems results

Theorem 7. Let $(M, F=\alpha+\beta)$ be a compact $n$-Randers space of constant flag curvature. The following statements hold:

(a) if $\operatorname{dim}(p(M, F))=\frac{n(n+1)}{2}$, then $\alpha$ is of constant sectional curvature;

(b) if $\operatorname{dim}(p(M, F))>\frac{n(n+1)}{2}$, then $F$ is a locally Minkowski metric.

Proof. Let us suppose $\operatorname{dim}(p(M, F))=\frac{n(n+1)}{2}$. Due to the discussions in the proof of Theorem 2 , in this case we have $p(M, F) \subseteq k(M, \alpha)$ and thus,

$$
\frac{n(n+1)}{2}=\operatorname{dim}(p(M, F)) \leq \operatorname{dim}(k(M, \alpha)) .
$$

Hence $(M, \alpha)$ is of maximum rank $\frac{n(n+1)}{2}$ and it is well-known that in this case $\alpha$ is of constant sectional curvature. This proves $(a)$. To prove $(b)$, we notice that if $\operatorname{dim}(p(M, F))>\frac{n(n+1)}{2}$, then we must have $s^{i}{ }_{j}=0$, Otherwise, by proof of Theorem 2 , we have $\operatorname{dim}(p(M, F)) \leq \frac{n(n+1)}{2}$. Now, $s^{i}{ }_{j}=0$ and $\mathbf{S}=0$ results that $F$ is a Berwald metric which is already of constant flag curvature. $F$ is not Riemannian and Numata's theorem ensures that $\mathbf{K}=0$. Finally, AkbarZadeh's classification theorem entails $F$ is a locally Minkowski metric.

Example 3. In [6], the authors presented a worthily source of a 1-parameter family of Randers metric $F_{\kappa}=\alpha_{\kappa}+\beta_{\kappa}$ on the Lie group $S^{3}$ which all are of constant positive flag curvature $\kappa$. Due to their construction, non of the Riemannian metrics $\alpha_{\kappa}$ is of constant sectional curvature and hence, by Theorem 7 , it follows that $\operatorname{dim}\left(p\left(S^{3}, F_{\kappa}\right)\right)<6$.

\section{The Lorentz nonlinear connection and Randers projective symmetry}

The stage on which special relativity is played out is a specific four dimensional manifold, known as Minkowski spacetime. The $x^{\mu}, \mu=0,1,2,3$, are coordinates on this manifold and conventionally, we set $x^{0}=t$. The elements of spacetime are known as events; an event is specified by giving its location in both space and time. The infinitesimal (distance) between two points known as the spacetime interval is defined by

$$
d s^{2}=\eta_{\mu \nu} d x^{\mu} d x^{\nu}=-d t^{2}+d x^{2}+d y^{2}+d z^{2} .
$$


The matrices $\Lambda$ satisfying $\Lambda^{T} \eta \Lambda=\eta$ are known as the Lorentz transformations. As a notable well-known case, consider the celebrated Randers metric of the form $F=\sqrt{\eta_{\mu \nu} d x^{\mu} d x^{\nu}}+\mathbf{A}_{i} d x^{i}$ on the 4-manifold of spacetime, where $\mathbf{A}$ is the electromagnetic vectorial potential and $\mathbf{F}=d \mathbf{A}$ obtained in the Cartesian coordinates $(t, x, y, z)$ as

$$
\mathbf{F}_{\mu \nu}=\left(\begin{array}{cccc}
0 & -E_{x} & -E_{y} & E_{z} \\
E_{x} & 0 & B_{z} & -B_{y} \\
E_{y} & -B_{z} & 0 & B_{x} \\
E_{z} & B_{y} & -B_{x} & 0
\end{array}\right)
$$

Notice that $\mathbf{F}=0$ if and only if $F$ is locally projectively flat. Hence, the presence of a proper electromagnetic field, entails non-locally projectively flatness of $F$. Consider a Lorentz transformation $\Lambda$ which maps the coordinates $(t, x, y, z)$ onto $(\bar{t}, \bar{x}, \bar{y}, \bar{z})$. $\Lambda$ changes $F=\sqrt{\eta_{\mu \nu} d x^{\mu} d x^{\nu}}+$ $\mathbf{A}_{i} d x^{i}$ to $\bar{F}=\sqrt{\eta_{\mu \nu} d x^{\mu} d x^{\nu}}+\overline{\mathbf{A}}_{i} d x^{i}$. Following an extensive discussion on projectively related Randers metrics in [20], we conclude that the Lorentz transformation $\Lambda$ is $F$-projective if and only if $\Lambda^{T} \mathbf{F} \Lambda=\mathbf{F}$. The collection of all such Lorentz transformation forms a subgroup of the Lorentz group which is at once a subgroup of projective group $P(M, F)$. Theory of Finsler spaces with $(\alpha, \beta)$-metrics was studied by such famous geometers as M. Matsumoto, D. Bao and many others as a natural extension of the theory of Randers spaces. Associated to any $(\alpha, \beta)$-metric $F=F(\alpha, \beta)$ one may consider a nonlinear connection called Lorentz connection which has physical applications in the study of gravitational and electromagnetic fields [12]. In this section, we uncover some results about its projective symmetry in a Finsler space with a Randers metric.

Let $F=F(\alpha, \beta)$ be an $(\alpha, \beta)$-metric on the manifold $M$. Through a variational approach, Lorentz equations are derived using Euler-Lagrange equations in the following form:

$$
\frac{d^{2} x^{i}}{d s^{2}}+\Gamma_{j k}^{i} \frac{d x^{j}}{d s} \frac{d x^{k}}{d s}+\sigma\left(x, \frac{d x}{d s}\right) s^{i}{ }_{j} \frac{d x^{j}}{d s}=0
$$

where $d s^{2}=\alpha^{2}\left(x, \frac{d x}{d t}\right) d t^{2}$ and $\sigma=\alpha F_{\beta}^{2} / F_{\alpha}^{2}$. The Lorentz nonlinear connection $\stackrel{\circ}{G}_{j}^{i}$ is now defined by

$$
\stackrel{\circ}{G}_{j}^{i}(x, y)=\Gamma_{j k}^{i}(x) y^{k}+\sigma(x, y) s^{i}{ }_{j} .
$$

Every geometric object associated to the Lorentz connection will be denoted by the sign "oo" on top. Notice that the Lorentz nonlinear connection is determined by the Finsler-Lagrange metric $F=F(\alpha, \beta)$ only. Notice that the autoparallel curves of the nonlinear connection $\stackrel{\circ}{G}_{j}^{i}$, in the natural parameterizations (i.e. $\alpha\left(x, \frac{d x}{d s}\right)=1$ ), coincide with the integral curves of the canonical semispray $S$ given by

$$
S=y^{i} \frac{\partial}{\partial x^{i}}-2 \stackrel{\circ}{G^{i}} \frac{\partial}{\partial y^{i}}, \quad \text { where } \quad 2 \stackrel{\circ}{G}^{i}=\Gamma_{j k}^{i} y^{j} y^{k}+\alpha s_{\circ}^{i} .
$$

Akbar-Zadeh in [4] considers the Berwald connection of the semispray $\stackrel{\circ}{G}^{i}$ to obtain a covariant derivative and derived a unified formulation for electromagnetism and gravity. However, it encounters physical consistency: all the formulation require being invariant under the Lorentz group in flat space-time. This is not satisfied generally. It can be shown that the only Lorentz transformation which preserve the spray $\stackrel{\circ}{G}^{i}$ are those satisfying $\Lambda^{T} \mathbf{F} \Lambda=\mathbf{F}$. 


\section{Acknowledgements}

The authors would like to express their grateful thanks to the referees for their valuable comments. This work was supported in part by the Institute for Research in Fundamental Sciences (IPM) by the grant No. 89530036. The first author wishes to thank Borzoo Nazari for many fruitful conversations.

\section{References}

[1] Akbar-Zadeh H., Champs de vecteurs projectifs sur le fibré unitaire, J. Math. Pures Appl. (9) 65 (1986), $47-79$.

[2] Akbar-Zadeh H., Sur les espaces de Finsler à courbures sectionelles constantes, Acad. Roy. Belg. Bull. Cl. Sci. (5) 74 (1988), no. 10, 281-322.

[3] Akbar-Zadeh H., Espaces à tenseurs de Ricci parallèle admettant des transformations projectives, Rend. Mat. (6) 11 (1978), 85-96.

[4] Akbar-Zadeh H., Generalized Einstein manifolds, J. Geom. Phys. 17 (1995), 342-380.

[5] Bao D., Robles C., On Randers metrics of constant curvature, Rep. Math. Phys. 51 (2003), 9-42.

[6] Bao D., Shen Z., Finsler metrics of constant positive curvature on the Lie group $S^{3}$, J. Lond. Math. Soc. 66 (2002), 453-467.

[7] Barnes A., Projective collineations in Einstein spaces, Classical Quantum Gravity 10 (1993), 1139-1145.

[8] Gibbons G.W., Herdeiro C.A.R., Warnick C.M., Werner M.C., Stationary metrics and optical ZermeloRanders-Finsler geometry, Phys. Rev. D 79 (2009), 044022, 21 pages, arXiv:0811.2877.

[9] Hall G.S., Lonie D.P., The principle of equivalence and cosmological metrics, J. Math. Phys. 49 (2008), 022502, 13 pages.

[10] Hiramato H., Riemannian manifolds admitting a projective vector field, Kodai Math. J. 3 (1980), 397-406.

[11] Israel I., Relativity, astrophysics and cosmology, Dordrecht, Boston, 1973.

[12] Miron R., Finsler-Lagrange spaces with ( $\alpha, \beta)$-metrics and Ingarden spaces, Rep. Math. Phys. 58 (2006), 417-431.

[13] Mo X., On the non-Riemannian quantity $H$ of a Finsler metric, Differential. Geom. Appl. 27 (2009), 7-14.

[14] Mo X., A global classification result for Randers metrics of scalar curvature on closed manifolds, Nonlinear Anal. 69 (2008), 2996-3004.

[15] Najafi B., Shen Z., Tayebi A., Finsler metrics of scalar flag curvature with special non-Riemannian curvature properties, Geom. Dedicata 131 (2008), 87-97.

[16] Najafi B., Tayebi A., A new quantity in Finsler geometry, C. R. Math. Acad. Sci. Paris 349 (2011), 81-83.

[17] Obata M., Certain conditions for a Riemannian manifold to be isometric with a sphere, J. Math. Soc. Japan. 14 (1962), 333-340.

[18] Randers G., On an asymmetric metric in the four-space of general relativity, Phys. Rev. 59 (1941), 195-199.

[19] Robles C., Einstein metrics of Randers type, Ph.D. thesis, University of British Columbia, Canada, 2003.

[20] Shen Y., Yu Y., On projectively related Randers metric, Internat. J. Math. 19 (2008), 503-520.

[21] Shen Z., Differential geometry of spray and Finsler spaces, Kluwer Academic Publishers, Dordrecht, 2001.

[22] Shen Z., Projectively flat Finsler metrics of constant flag curvature, Trans. Amer. Math. Soc. 355 (2002), $1713-1728$.

[23] Shen Z.M., Xing H., On Randers metrics of isotropic S-curvature, Acta Math. Sin. (Engl. Ser.) 24 (2008), 789-796.

[24] Stavrinos P.C., Gravitational and cosmological considerations based on the Finsler and Lagrange metric structures, Nonlinear Anal. 71 (2009), e1380-e1392.

[25] Tanno S., Some differential equations on Riemannian manifolds, J. Math. Soc. Japan 30 (1978), 509-531.

[26] Tashiro Y., Complete Riemannian manifolds and some vector fields, Trans. Amer. Math. Soc. 117 (1965), 251-275.

[27] Yamauchi K., On infinitesimal projective transformations of a Riemannian manifold with constant scalar curvature, Hokkaido. Math. J. 8 (1979), 167-175.

[28] Yano K., The theory of Lie derivatives and its applications, North Holland, Amsterdam, 1957. 